\title{
On Proximal Relations in Transformation Semigroups Arising from Generalized Shifts
}

\author{
Fatemah Ayatollah Zadeh Shirazi ${ }^{1, *}$, Amir Fallahpour ${ }^{2}$, \\ Mohammad Reza Mardanbeigi ${ }^{3}$ and Zahra Nili Ahmadabadi ${ }^{3}$ \\ ${ }^{1}$ Faculty of Mathematics Statistics and Computer Science, College of Science, \\ University of Tehran, Enghelab Ave., Tehran, Iran \\ ${ }^{2}$ Faculty of Mathematics and Computer Science, College of Science, Amirkabir \\ University of Technology, Tehran, Iran \\ ${ }^{3}$ Islamic Azad University, Science and Research Branch Tehran, Iran
}

Received 16 October 2017; Accepted (in revised version) 24 September 2019

\begin{abstract}
For a finite discrete topological space $X$ with at least two elements, a nonempty set $\Gamma$, and a map $\varphi: \Gamma \rightarrow \Gamma, \sigma_{\varphi}: X^{\Gamma} \rightarrow X^{\Gamma}$ with $\sigma_{\varphi}\left(\left(x_{\alpha}\right)_{\alpha \in \Gamma}\right)=\left(x_{\varphi(\alpha)}\right)_{\alpha \in \Gamma}$ (for $\left(x_{\alpha}\right)_{\alpha \in \Gamma} \in X^{\Gamma}$ ) is a generalized shift. In this text for $\mathcal{S}=\left\{\sigma_{\psi}: \psi \in \Gamma^{\Gamma}\right\}$ and $\mathcal{H}=\left\{\sigma_{\psi}: \Gamma \stackrel{\psi}{\rightarrow} \Gamma\right.$ is bijective $\}$ we study proximal relations of transformation semigroups $\left(\mathcal{S}, X^{\Gamma}\right)$ and $\left(\mathcal{H}, X^{\Gamma}\right)$. Regarding proximal relation we prove:

$$
P\left(\mathcal{S}, X^{\Gamma}\right)=\left\{\left(\left(x_{\alpha}\right)_{\alpha \in \Gamma},\left(y_{\alpha}\right)_{\alpha \in \Gamma}\right) \in X^{\Gamma} \times X^{\Gamma}: \exists \beta \in \Gamma\left(x_{\beta}=y_{\beta}\right)\right\}
$$

and $P\left(\mathcal{H}, X^{\Gamma}\right) \subseteq\left\{\left(\left(x_{\alpha}\right)_{\alpha \in \Gamma},\left(y_{\alpha}\right)_{\alpha \in \Gamma}\right) \in X^{\Gamma} \times X^{\Gamma}:\left\{\beta \in \Gamma: x_{\beta}=y_{\beta}\right\}\right.$ is infinite $\} \cup$ $\{(x, x): x \in \mathcal{X}\}$.

Moreover, for infinite $\Gamma$, both transformation semigroups $\left(\mathcal{S}, X^{\Gamma}\right)$ and $\left(\mathcal{H}, X^{\Gamma}\right)$ are regionally proximal, i.e., $Q\left(\mathcal{S}, X^{\Gamma}\right)=Q\left(\mathcal{H}, X^{\Gamma}\right)=X^{\Gamma} \times X^{\Gamma}$, also for sydetically proximal relation we have $L\left(\mathcal{H}, X^{\Gamma}\right)=\left\{\left(\left(x_{\alpha}\right)_{\alpha \in \Gamma},\left(y_{\alpha}\right)_{\alpha \in \Gamma}\right) \in X^{\Gamma} \times X^{\Gamma}:\left\{\gamma \in \Gamma: x_{\gamma} \neq y_{\gamma}\right\}\right.$ is finite\}.
\end{abstract}

Key Words: Generalized shift, proximal relation, transformation semigroup.

AMS Subject Classifications: 54H15, 37B09

\section{Preliminaries}

By a (left topological) transformation semigroup $(S, Z, \pi)$ or simply $(S, Z)$ we mean a compact Hausdorff topological space $Z$ (phase space), discrete topological semigroup $S$

*Corresponding author. Email addresses: fatemah@khayam.ut.ac.ir, f.a.z.shirazi@ut.ac.ir (F. Ayatollah Zadeh Shirazi), amir.falah90@yahoo.com (A. Fallahpour), mrmardanbeigi@srbiau.ac.ir (M. Mardanbeigi), zahra.nili.a@gmail.com (Z. Nili Ahmadabadi) 
(phase semigroup) with identity $e$ and continuous map $\pi: S \times Z \rightarrow Z(\pi(s, z)=s z, s \in$ $S, z \in Z$ ) such that for all $z \in Z$ and $s, t \in S$ we have $e z=z$, $(s t) z=s(t z)$. If $S$ is a discrete topological group too, then we call the transformation semigroup $(S, Z)$, a transformation group. We say $(x, y) \in Z \times Z$ is a proximal pair of $(S, Z)$ if there exists a net $\left\{s_{\lambda}\right\}_{\lambda \in \Lambda}$ in $S$ with

$$
\lim _{\lambda \in \Lambda} s_{\lambda} x=\lim _{\lambda \in \Lambda} s_{\lambda} y \text {. }
$$

We denote the collection of all proximal pairs of $(S, Z)$ by $P(S, Z)$ and call it proximal relation on $(S, Z)$, for more details on proximal relations we refer the interested reader to $[4,8]$.

In the transformation semigroup $(S, Z)$ we call $(x, y) \in Z \times Z$ a regionally proximal pair if there exists a net $\left\{\left(s_{\lambda}, x_{\lambda}, y_{\lambda}\right)\right\}_{\lambda \in \Lambda}$ in $S \times Z \times Z$ such that

$$
\lim _{\lambda \in \Lambda} x_{\lambda}=x, \quad \lim _{\lambda \in \Lambda} y_{\lambda}=y \quad \text { and } \quad \lim _{\lambda \in \Lambda} s_{\lambda} x_{\lambda}=\lim _{\lambda \in \Lambda} s_{\lambda} y_{\lambda}
$$

We denote the collection of all regionally proximal pairs of $(S, Z)$ by $Q(S, Z)$ and call it regionally proximal relation on $(S, Z)$. Obviously we have $P(S, Z) \subseteq Q(S, Z)$. In the transformation group $(T, Z)$, by [9] we call $L(T, Z)=\{(x, y) \in Z \times Z: \overline{T(x, y)} \subseteq P(T, Z)\}$ the syndetically proximal relation of $(T, Z)$ (for details on the interaction of $L(T, Z), Q(T, Z)$ and $P(T, Z)$ with uniform structure of $Z$ see $[5,6,9])$.

\subsection{A collection of generalized shifts as phase semigroup}

For nonempty sets $X, \Gamma$ and self-map $\varphi: \Gamma \rightarrow \Gamma$ define the generalized shift $\sigma_{\varphi}: X^{\Gamma} \rightarrow X^{\Gamma}$ by $\sigma_{\varphi}\left(\left(x_{\alpha}\right)_{\alpha \in \Gamma}\right)=\left(x_{\varphi(\alpha)}\right)_{\alpha \in \Gamma}\left(\left(x_{\alpha}\right)_{\alpha \in \Gamma} \in X^{\Gamma}\right)$. Generalized shifts have been introduced for the first time in [2], in addition dynamical and non-dynamical properties of generalized shifts have been studied in several texts like [3] and [7]. It's well-known that if $X$ has a topological structure, then $\sigma_{\varphi}: X^{\Gamma} \rightarrow X^{\Gamma}$ is continuous (when $X^{\Gamma}$ equipped with product topology), in addition If $X$ has at least two elements, then $\sigma_{\varphi}: X^{\Gamma} \rightarrow X^{\Gamma}$ is a homeomorphism if and only if $\varphi: \Gamma \rightarrow \Gamma$ is bijective.

Convention. In this text suppose $X$ is a finite discrete topological space with at least two elements, $\Gamma$ is a nonempty set, $\mathcal{X}:=X^{\Gamma}$, and:

- $\mathcal{S}:=\left\{\sigma_{\varphi}: \varphi \in \Gamma^{\Gamma}\right\}$, is the semigroup of generalized shifts on $X^{\Gamma}$,

- $\mathcal{H}:=\left\{\sigma_{\varphi}: \varphi \in \Gamma^{\Gamma}\right.$ and $\varphi: \Gamma \rightarrow \Gamma$ is bijective $\}$, is the group of generalized shift homeomorphisms on $X^{\Gamma}$.

Equip $X^{\Gamma}$ with product (pointwise convergence) topology. Now we may consider $\mathcal{S}$ (resp. $\mathcal{H}$ ) as a subsemigroup (resp. subgroup) of continuous maps (resp. homeomorphisms) from $\mathcal{X}$ to itself, so $\mathcal{S}$ (resp. $\mathcal{H}$ ) acts on $\mathcal{X}$ in a natural way.

Our aim in this text is to study $P(T, \mathcal{X}), Q(T, \mathcal{X})$, and $L(T, \mathcal{X})$ for $T=\mathcal{H}, \mathcal{S}$. Readers interested in this subject may refer to [1] too. 


\section{Proximal and regionally proximal relations of $(\mathcal{S}, \mathcal{X})$}

In this section we prove that

$$
\begin{aligned}
& P(\mathcal{S}, \mathcal{X})=\left\{\left(\left(x_{\alpha}\right)_{\alpha \in \Gamma},\left(y_{\alpha}\right)_{\alpha \in \Gamma}\right) \in \mathcal{X} \times \mathcal{X}: \exists \beta \in \Gamma\left(x_{\beta}=y_{\beta}\right)\right\}, \\
& Q(\mathcal{S}, \mathcal{X})= \begin{cases}\mathcal{X} \times \mathcal{X}, \quad \Gamma \text { is infinite, } \\
P(\mathcal{S}, \mathcal{X}), \quad \Gamma \text { is finite. }\end{cases}
\end{aligned}
$$

Theorem 2.1. $P(\mathcal{S}, \mathcal{X})=\left\{\left(\left(x_{\alpha}\right)_{\alpha \in \Gamma},\left(y_{\alpha}\right)_{\alpha \in \Gamma}\right) \in \mathcal{X} \times \mathcal{X}: \exists \beta \in \Gamma\left(x_{\beta}=y_{\beta}\right)\right\}$.

Proof. First consider $\beta \in \Gamma$ and $\left(x_{\alpha}\right)_{\alpha \in \Gamma},\left(y_{\alpha}\right)_{\alpha \in \Gamma} \in \mathcal{X}$ by $x_{\beta}=y_{\beta}$. Define $\psi: \Gamma \rightarrow \Gamma$ with $\psi(\alpha)=\beta$ for all $\alpha \in \Gamma$. Then

$$
\begin{aligned}
& \sigma_{\psi}\left(\left(x_{\alpha}\right)_{\alpha \in \Gamma}\right)=\left(x_{\beta}\right)_{\alpha \in \Gamma}=\left(y_{\beta}\right)_{\alpha \in \Gamma}=\sigma_{\psi}\left(\left(y_{\alpha}\right)_{\alpha \in \Gamma}\right), \\
& \left(\left(x_{\alpha}\right)_{\alpha \in \Gamma},\left(y_{\alpha}\right)_{\alpha \in \Gamma}\right) \in P(\mathcal{S}, \mathcal{X}) .
\end{aligned}
$$

Conversely, suppose $\left(\left(x_{\alpha}\right)_{\alpha \in \Gamma},\left(y_{\alpha}\right)_{\alpha \in \Gamma}\right) \in P(\mathcal{S}, \mathcal{X})$. There exists a net $\left\{\sigma_{\varphi_{\lambda}}\right\}_{\lambda \in \Lambda}$ in $\mathcal{S}$ with

$$
\lim _{\lambda \in \Lambda} \sigma_{\varphi_{\lambda}}\left(\left(x_{\alpha}\right)_{\alpha \in \Gamma}\right)=\lim _{\lambda \in \Lambda} \sigma_{\varphi_{\lambda}}\left(\left(y_{\alpha}\right)_{\alpha \in \Gamma}\right)=:\left(z_{\alpha}\right)_{\alpha \in \Gamma} .
$$

Choose arbitrary $\theta \in \Gamma$, then

$$
\lim _{\lambda \in \Lambda} x_{\varphi_{\lambda}(\theta)}=\lim _{\lambda \in \Lambda} y_{\varphi_{\lambda}(\theta)}=z_{\theta}
$$

in $X$. Since $X$ is discrete, there exists $\lambda_{0} \in \Lambda$ such that $x_{\varphi_{\lambda}(\theta)}=y_{\varphi_{\lambda}(\theta)}=z_{\theta}$ for all $\lambda \geq \lambda_{0}$, in particular for $\beta=\varphi_{\lambda_{0}(\theta)}$ we have $x_{\beta}=y_{\beta}$.

Lemma 2.1. For infinite $\Gamma$ we have: $Q(\mathcal{S}, \mathcal{X})=Q(\mathcal{H}, \mathcal{X})=\mathcal{X} \times \mathcal{X}$.

Proof. Suppose $\Gamma$ is infinite, then there exits a bijection $\mu: \Gamma \times \mathbb{Z} \rightarrow \Gamma$, in particular $\{\mu(\{\alpha\} \times \mathbb{Z}): \alpha \in \Gamma\}$ is a partition of $\Gamma$ to its infinite countable subsets. Define bijection $\varphi: \Gamma \rightarrow \Gamma$ by $\varphi(\mu(\alpha, n))=\mu(\alpha, n+1)$ for all $\alpha \in \Gamma$ and $n \in \mathbb{Z}$. Consider $p \in X$ and $\left(x_{\alpha}\right)_{\alpha \in \Gamma},\left(y_{\alpha}\right)_{\alpha \in \Gamma} \in \mathcal{X}$. For all $n \geq 1$ and $\alpha \in \Gamma$ let:

$$
\begin{aligned}
& x_{\alpha}^{n}:= \begin{cases}x_{\alpha}, & \alpha=\mu(\beta, k) \text { for some } \beta \in \Gamma \text { and } k \leq n, \\
p, & \text { otherwise, }\end{cases} \\
& y_{\alpha}^{n}:= \begin{cases}y_{\alpha}, & \alpha=\mu(\beta, k) \text { for some } \beta \in \Gamma \text { and } k \leq n, \\
p, & \text { otherwise, }\end{cases}
\end{aligned}
$$

then:

$$
\begin{aligned}
& \lim _{n \rightarrow+\infty}\left(x_{\alpha}^{n}\right)_{\alpha \in \Gamma}=\left(x_{\alpha}\right)_{\alpha \in \Gamma,} \\
& \lim _{n \rightarrow+\infty}\left(y_{\alpha}^{n}\right)_{\alpha \in \Gamma}=\left(y_{\alpha}\right)_{\alpha \in \Gamma}, \\
& \lim _{n \rightarrow \infty} \sigma_{\varphi^{2 n}}\left(\left(x_{\alpha}^{n}\right)_{\alpha \in \Gamma}\right)=\left(p_{\alpha}\right)_{\alpha \in \Gamma}=\lim _{n \rightarrow+\infty} \sigma_{\varphi^{2 n}}\left(\left(y_{\alpha}^{n}\right)_{\alpha \in \Gamma}\right) .
\end{aligned}
$$

By $\sigma_{\varphi^{2 n}} \in \mathcal{H}$ for all $n \geq 1$ and using the above statements, we have $\left(\left(x_{\alpha}\right)_{\alpha \in \Gamma},\left(y_{\alpha}\right)_{\alpha \in \Gamma}\right) \in$ $Q(\mathcal{H}, \mathcal{X}) \subseteq Q(\mathcal{S}, \mathcal{X})$ 
Lemma 2.2. For finite $\Gamma$ and any subsemigroup $\mathcal{T}$ of $\mathcal{S}$ we have $Q(\mathcal{T}, \mathcal{X})=P(\mathcal{T}, \mathcal{X})$.

Proof. We must only prove $Q(\mathcal{T}, \mathcal{X}) \subseteq P(\mathcal{T}, \mathcal{X})$. Suppose $(x, y) \in Q(\mathcal{T}, \mathcal{X})$, then there exists a net $\left\{\left(x_{\lambda}, y_{\lambda}, t_{\lambda}\right)\right\}_{\lambda \in \Lambda}$ in $\mathcal{X} \times \mathcal{X} \times \mathcal{T}$ such that

$$
\begin{aligned}
& \lim _{\lambda \in \Lambda} x_{\lambda}=x, \quad \lim _{\lambda \in \Lambda} y_{\lambda}=y, \\
& \lim _{\lambda \in \Lambda} t_{\lambda} x_{\lambda}=\lim _{\lambda \in \Lambda} t_{\lambda} y_{\lambda}=: z .
\end{aligned}
$$

Since $\mathcal{X} \times \mathcal{X} \times \mathcal{T}$ is finite, $\left\{\left(x_{\lambda}, y_{\lambda}, t_{\lambda}\right)\right\}_{\lambda \in \Lambda}$ has a constant subnet like $\left\{\left(x_{\lambda_{\mu}}, y_{\lambda_{\mu}}, t_{\lambda_{\mu}}\right)\right\}_{\mu \in M}$, so there exists $t \in \mathcal{T}$ such that for all $\mu \in M$ we have $x=x_{\lambda_{\mu}}, y=y_{\lambda_{\mu}}$ and $t=t_{\lambda_{\mu}}$, therefore $t x=t y(=z)$ and $(x, y) \in P(\mathcal{T}, \mathcal{X})$.

Theorem 2.2. We have:

$$
Q(\mathcal{S}, \mathcal{X})= \begin{cases}\mathcal{X} \times \mathcal{X}, & \Gamma \text { is infinite } \\ P(\mathcal{S}, \mathcal{X}), & \Gamma \text { is finite. }\end{cases}
$$

Proof. Use Lemmas 2.1 and 2.2.

\section{Proximal and regionally proximal relations of $(\mathcal{H}, \mathcal{X})$}

Note that for finite $\Gamma, \mathcal{H}$ is a finite subset of homeomorphisms on $\mathcal{X}$ and $P(\mathcal{H}, \mathcal{X})=$ $\{(x, x): x \in \mathcal{X}\}$, also using Lemmas 2.1 and 2.2 we have:

$$
Q(\mathcal{H}, \mathcal{X})= \begin{cases}\mathcal{X} \times \mathcal{X} \\ P(\mathcal{H}, \mathcal{X})=\{(x, x): x \in \mathcal{X}\}, & \Gamma \text { is infinite }\end{cases}
$$

In this section we show that:

$$
\left\{\left(\left(x_{\alpha}\right)_{\alpha \in \Gamma,}\left(y_{\alpha}\right)_{\alpha \in \Gamma}\right): \max \left(\operatorname{card}\left(\left\{\beta \in \Gamma: x_{\beta} \neq y_{\beta}\right\}\right), \aleph_{0}\right) \leq \operatorname{card}\left(\left\{\beta \in \Gamma: x_{\beta}=y_{\beta}\right\}\right)\right\}
$$

is a subset of $P(\mathcal{H}, \mathcal{X})$, which is a subset of

$$
\left\{\left(\left(x_{\alpha}\right)_{\alpha \in \Gamma},\left(y_{\alpha}\right)_{\alpha \in \Gamma}\right) \in \mathcal{X} \times \mathcal{X}:\left\{\beta \in \Gamma: x_{\beta}=y_{\beta}\right\} \text { is infinite }\right\} \cup\{(x, x): x \in \mathcal{X}\}
$$

in its turn. In particular, for countable $\Gamma$ we prove

$P(\mathcal{H}, \mathcal{X})=\left\{\left(\left(x_{\alpha}\right)_{\alpha \in \Gamma},\left(y_{\alpha}\right)_{\alpha \in \Gamma}\right) \in \mathcal{X} \times \mathcal{X}:\left\{\beta \in \Gamma: x_{\beta}=y_{\beta}\right\}\right.$ is infinite $\} \cup\{(x, x): x \in \mathcal{X}\}$.

Lemma 3.1. For infinite $\Gamma$, we have:

$$
P(\mathcal{H}, \mathcal{X}) \subseteq\left\{\left(\left(x_{\alpha}\right)_{\alpha \in \Gamma},\left(y_{\alpha}\right)_{\alpha \in \Gamma}\right) \in \mathcal{X} \times \mathcal{X}:\left\{\beta \in \Gamma: x_{\beta}=y_{\beta}\right\} \text { is infinite }\right\}
$$


Proof. Consider $\left(\left(x_{\alpha}\right)_{\alpha \in \Gamma},\left(y_{\alpha}\right)_{\alpha \in \Gamma}\right) \in P(\mathcal{H}, \mathcal{X})$, then there exists a net $\left\{\sigma_{\varphi_{\lambda}}\right\}_{\lambda \in \Lambda}$ in $\mathcal{H}$ with

$$
\lim _{\lambda \in \Lambda} \sigma_{\varphi_{\lambda}}\left(\left(x_{\alpha}\right)_{\alpha \in \Gamma}\right)=\lim _{\lambda \in \Lambda} \sigma_{\varphi_{\lambda}}\left(\left(y_{\alpha}\right)_{\alpha \in \Gamma}\right)=:\left(z_{\alpha}\right)_{\alpha \in \Gamma} .
$$

Choose distinct $\theta_{1}, \cdots, \theta_{n} \in \Gamma$. For all $i \in\{1, \cdots, n\}$ we have

$$
\lim _{\lambda \in \Lambda} x_{\varphi_{\lambda}\left(\theta_{i}\right)}=\lim _{\lambda \in \Lambda} y_{\varphi_{\lambda}\left(\theta_{i}\right)}=z_{\theta_{i}} \quad \text { in } X,
$$

so there exists $\lambda_{1}, \cdots, \lambda_{n} \in \Lambda$ with $x_{\varphi_{\lambda}\left(\theta_{i}\right)}=y_{\varphi_{\lambda}\left(\theta_{i}\right)}=z_{\theta_{i}}$ for all $\lambda \geq \lambda_{i}$. There exists $\mu \in \Lambda$ with $\mu \geq \lambda_{1}, \cdots, \lambda_{n}$, thus $x_{\varphi_{\mu}\left(\theta_{i}\right)}=y_{\varphi_{\mu}\left(\theta_{i}\right)}$ for $i=1, \cdots, n$. Since $\varphi_{\mu}: \Gamma \rightarrow \Gamma$ is bijective and $\theta_{1}, \cdots, \theta_{n}$ are pairwise distinct, $\left\{\varphi_{\mu}\left(\theta_{1}\right), \cdots, \varphi_{\mu}\left(\theta_{n}\right)\right\}$ has exactly $n$ elements and $\left\{\varphi_{\mu}\left(\theta_{1}\right), \cdots, \varphi_{\mu}\left(\theta_{n}\right)\right\} \subseteq\left\{\beta \in \Gamma: x_{\beta}=y_{\beta}\right\}$. Hence $\left\{\beta \in \Gamma: x_{\beta}=y_{\beta}\right\}$ has at least $n$ elements (for all $n \geq 1$ ) and it is infinite.

Theorem 3.1. We have:

$$
\begin{gathered}
P(\mathcal{H}, \mathcal{X}) \subseteq\left\{\left(\left(x_{\alpha}\right)_{\alpha \in \Gamma},\left(y_{\alpha}\right)_{\alpha \in \Gamma}\right) \in \mathcal{X} \times \mathcal{X}:\left\{\beta \in \Gamma: x_{\beta}=y_{\beta}\right\} \text { is infinite }\right\} \\
\cup\{(x, x): x \in \mathcal{X}\} .
\end{gathered}
$$

Proof. Use Lemma 3.1 and the fact that for finite $\Gamma, \mathcal{H}$ is a finite subset of homeomorphisms on $\mathcal{X}$. So for finite $\Gamma$ we have $P(\mathcal{H}, \mathcal{X})=\{(w, w): w \in \mathcal{X}\}$.

Lemma 3.2. For infinite countable $\Gamma$, we have

$$
P(\mathcal{H}, \mathcal{X})=\left\{\left(\left(x_{\alpha}\right)_{\alpha \in \Gamma},\left(y_{\alpha}\right)_{\alpha \in \Gamma}\right) \in \mathcal{X} \times \mathcal{X}:\left\{\beta \in \Gamma: x_{\beta}=y_{\beta}\right\} \text { is infinite }\right\} .
$$

Proof. Using Lemma 3.1 we must only prove:

$$
P(\mathcal{H}, \mathcal{X}) \supseteq\left\{\left(\left(x_{\alpha}\right)_{\alpha \in \Gamma},\left(y_{\alpha}\right)_{\alpha \in \Gamma}\right) \in \mathcal{X} \times \mathcal{X}:\left\{\beta \in \Gamma: x_{\beta}=y_{\beta}\right\} \text { is infinite }\right\} .
$$

Consider $\left(x_{\alpha}\right)_{\alpha \in \Gamma},\left(y_{\alpha}\right)_{\alpha \in \Gamma} \in \mathcal{X}$ with infinite set $\left\{\beta \in \Gamma: x_{\beta}=y_{\beta}\right\}=\left\{\beta_{1}, \beta_{2}, \cdots\right\}$ and distinct $\beta_{i}$ s. Also suppose $\Gamma=\left\{\alpha_{1}, \alpha_{2}, \cdots\right\}$ with distinct $\alpha_{i}$ s. For all $n \geq 1$ there exists bijection $\varphi_{n}: \Gamma \rightarrow \Gamma$ with $\varphi_{n}\left(\alpha_{i}\right)=\beta_{i}$ for $i \in\{1, \cdots, n\}$. Let $\alpha \in \Gamma$, there exists $i \geq 1$ with $\alpha=\alpha_{i}$. Since for all $n \geq i$ we have

$$
x_{\varphi_{n}(\alpha)}=x_{\varphi_{n}\left(\alpha_{i}\right)}=x_{\beta_{i}}=y_{\beta_{i}}=y_{\varphi_{n}\left(\alpha_{i}\right)}=y_{\varphi_{n}(\alpha)},
$$

we have

$$
\lim _{n \rightarrow \infty} x_{\varphi_{n}(\alpha)}=\lim _{n \rightarrow \infty} y_{\varphi_{n}(\alpha)}
$$

Therefore

$$
\begin{aligned}
& \lim _{n \rightarrow \infty} \sigma_{\varphi_{n}}\left(\left(x_{\alpha}\right)_{\alpha \in \Gamma}\right)=\lim _{n \rightarrow \infty}\left(x_{\varphi_{n}(\alpha)}\right)_{\alpha \in \Gamma}=\lim _{n \rightarrow \infty}\left(y_{\varphi_{n}(\alpha)}\right)_{\alpha \in \Gamma}=\lim _{n \rightarrow \infty} \sigma_{\varphi_{n}}\left(\left(y_{\alpha}\right)_{\alpha \in \Gamma}\right), \\
& \left(\left(x_{\alpha}\right)_{\alpha \in \Gamma},\left(y_{\alpha}\right)_{\alpha \in \Gamma}\right) \in P(\mathcal{H}, \mathcal{X}) .
\end{aligned}
$$

Thus, we complete the proof. 
Theorem 3.2. For countable $\Gamma$,

$$
\begin{aligned}
P(\mathcal{H}, \mathcal{X})=\{( & \left.\left.\left(x_{\alpha}\right)_{\alpha \in \Gamma},\left(y_{\alpha}\right)_{\alpha \in \Gamma}\right) \in \mathcal{X} \times \mathcal{X}:\left\{\beta \in \Gamma: x_{\beta}=y_{\beta}\right\} \text { is infinite }\right\} \\
& \cup\{(x, x): x \in \mathcal{X}\} .
\end{aligned}
$$

Proof. First note that for finite $\Gamma, \mathcal{H}$ is finite and $P(\mathcal{H}, \mathcal{X})=\{(x, x): x \in \mathcal{X}\}$. Now use Lemma 3.2.

Lemma 3.3. For infinite $\Gamma$, we have:

$$
\left\{\left(\left(x_{\alpha}\right)_{\alpha \in \Gamma},\left(y_{\alpha}\right)_{\alpha \in \Gamma}\right): \operatorname{card}\left(\left\{\beta \in \Gamma: x_{\beta} \neq y_{\beta}\right\}\right) \leq \operatorname{card}\left(\left\{\beta \in \Gamma: x_{\beta}=y_{\beta}\right\}\right)\right\} \subseteq P(\mathcal{H}, \mathcal{X}) .
$$

In particular,

$$
\left\{\left(\left(x_{\alpha}\right)_{\alpha \in \Gamma},\left(y_{\alpha}\right)_{\alpha \in \Gamma}\right):\left\{\beta \in \Gamma: x_{\beta} \neq y_{\beta}\right\} \text { is finite }\right\} \subseteq P(\mathcal{H}, \mathcal{X}) .
$$

Proof. Suppose $\Gamma$ is infinite. For $\left(x_{\alpha}\right)_{\alpha \in \Gamma},\left(y_{\alpha}\right)_{\alpha \in \Gamma} \in \mathcal{X}$, let:

$$
A:=\left\{\alpha \in \Gamma: x_{\alpha}=y_{\alpha}\right\}, \quad B:=\left\{\alpha \in \Gamma: x_{\alpha} \neq y_{\alpha}\right\}
$$

with $\operatorname{card}(B) \leq \operatorname{card}(A)$. There exists a one to one map $\lambda: B \rightarrow A$. By $\operatorname{card}(\Gamma)=$ $\operatorname{card}(A)+\operatorname{card}(B)$ and $\operatorname{card}(B) \leq \operatorname{card}(A), A$ is infinite. Since $A$ is infinite, we have $\operatorname{card}(A)=\operatorname{card}(A) \aleph_{0}$ so there exists a bijection $\varphi: A \times \mathbb{N} \rightarrow A$. For all $\theta \in A$ let $K_{\theta}=\varphi(\{\theta\} \times \mathbb{N}) \cup \lambda^{-1}(\theta)$. Thus $K_{\theta}$ s are disjoint infinite countable subsets of $\Gamma$, as a matter of fact $\left\{K_{\theta}: \theta \in A\right\}$ is a partition of $\Gamma$ to some of its infinite countable subsets. For all $\theta \in A,\left\{\alpha \in K_{\theta}: x_{\alpha}=y_{\alpha}\right\}=\varphi(\{\theta\} \times \mathbb{N})$ is infinite and $K_{\theta}$ is infinite countable. By Lemma 3.2 there exists a sequence $\left\{\psi_{n}^{\theta}\right\}$ of permutations on $K_{\theta}$ such that

$$
\lim _{n \rightarrow \infty} \sigma_{\psi_{n}^{\theta}}\left(x_{\alpha}\right)_{\alpha \in K_{\theta}}=\lim _{n \rightarrow \infty} \sigma_{\psi_{n}^{\theta}}\left(y_{\alpha}\right)_{\alpha \in K_{\theta}} .
$$

For all $n \geq 1$ let

$$
\psi_{n}=\bigcup_{\theta \in A} \psi_{n}^{\theta}
$$

then $\psi_{n}: \Gamma \rightarrow \Gamma$ is bijective and

$$
\lim _{n \rightarrow \infty} \sigma_{\psi_{n}}\left(x_{\alpha}\right)_{\alpha \in \Gamma}=\lim _{n \rightarrow \infty} \sigma_{\psi_{n}}\left(y_{\alpha}\right)_{\alpha \in \Gamma},
$$

which completes the proof.

Theorem 3.3. The collection $\left\{\left(\left(x_{\alpha}\right)_{\alpha \in \Gamma},\left(y_{\alpha}\right)_{\alpha \in \Gamma}\right): \max \left(\operatorname{card}\left(\left\{\beta \in \Gamma: x_{\beta} \neq y_{\beta}\right\}\right), \aleph_{0}\right) \leq\right.$ $\left.\operatorname{card}\left(\left\{\beta \in \Gamma: x_{\beta}=y_{\beta}\right\}\right)\right\}$ is a subset of $P(\mathcal{H}, \mathcal{X})$.

Proof. If $\Gamma$ is finite, then

$$
\begin{aligned}
& \left\{\left(\left(x_{\alpha}\right)_{\alpha \in \Gamma},\left(y_{\alpha}\right)_{\alpha \in \Gamma}\right): \max \left(\operatorname{card}\left(\left\{\beta \in \Gamma: x_{\beta} \neq y_{\beta}\right\}\right), \aleph_{0}\right)\right. \\
\leq & \left.\operatorname{card}\left(\left\{\beta \in \Gamma: x_{\beta}=y_{\beta}\right\}\right)\right\}=\varnothing .
\end{aligned}
$$

Use Lemma 3.3 to complete the proof. 


\section{Syndetically proximal relations of $(\mathcal{H}, \mathcal{X})$}

In this section we prove:

$L(\mathcal{H}, \mathcal{X})= \begin{cases}\left.\left\{\left(x_{\alpha}\right)_{\alpha \in \Gamma},\left(y_{\alpha}\right)_{\alpha \in \Gamma}\right) \in \mathcal{X} \times \mathcal{X}:\left\{\gamma \in \Gamma: x_{\gamma} \neq y_{\gamma}\right\} \text { is finite }\right\}, & \Gamma \text { is infinite, } \\ \{(x, x): x \in \mathcal{X}\}, & \Gamma \text { is finite. }\end{cases}$

Lemma 4.1. For $\left(x_{\alpha}\right)_{\alpha \in \Gamma},\left(y_{\alpha}\right)_{\alpha \in \Gamma},\left(u_{\alpha}\right)_{\alpha \in \Gamma} \in \mathcal{X}$, and $p, q \in X$ let:

$$
z_{\alpha}:=\left\{\begin{array}{ll}
q, & x_{\alpha} \neq y_{\alpha}, \\
u_{\alpha}, & x_{\alpha}=y_{\alpha},
\end{array} \quad \text { and } \quad w_{\alpha}:= \begin{cases}p, & x_{\alpha} \neq y_{\alpha}, \\
u_{\alpha}, & x_{\alpha}=y_{\alpha} .\end{cases}\right.
$$

We have:

1). if $\left(\left(x_{\alpha}\right)_{\alpha \in \Gamma},\left(y_{\alpha}\right)_{\alpha \in \Gamma}\right) \in P(\mathcal{H}, \mathcal{X})$, then $\left(\left(z_{\alpha}\right)_{\alpha \in \Gamma},\left(w_{\alpha}\right)_{\alpha \in \Gamma}\right) \in P(\mathcal{H}, \mathcal{X})$,

2). if $\left(\left(x_{\alpha}\right)_{\alpha \in \Gamma},\left(y_{\alpha}\right)_{\alpha \in \Gamma}\right) \in L(\mathcal{H}, \mathcal{X})$, then $\left(\left(z_{\alpha}\right)_{\alpha \in \Gamma},\left(w_{\alpha}\right)_{\alpha \in \Gamma}\right) \in L(\mathcal{H}, \mathcal{X})$.

Proof. 1) Suppose $\left(\left(x_{\alpha}\right)_{\alpha \in \Gamma},\left(y_{\alpha}\right)_{\alpha \in \Gamma}\right) \in P(\mathcal{H}, \mathcal{X})$, then there exists a net $\left\{\sigma_{\varphi_{\lambda}}\right\}_{\lambda \in \Lambda}$ in $\mathcal{H}$ such that

$$
\lim _{\lambda \in \Lambda} \sigma_{\varphi_{\lambda}}\left(\left(x_{\alpha}\right)_{\alpha \in \Gamma}\right)=\lim _{\lambda \in \Lambda} \sigma_{\varphi_{\lambda}}\left(\left(y_{\alpha}\right)_{\alpha \in \Gamma}\right) .
$$

Thus

$$
\lim _{\lambda \in \Lambda}\left(\left(x_{\varphi_{\lambda}(\alpha)}\right)_{\alpha \in \Gamma}\right)=\lim _{\lambda \in \Lambda}\left(\left(y_{\varphi_{\lambda}(\alpha)}\right)_{\alpha \in \Gamma}\right),
$$

i.e., for all $\alpha \in \Gamma$ there exists $\mathcal{K}_{\alpha} \in \Lambda$ such that:

$$
\forall \lambda \geq \kappa_{\alpha}\left(x_{\varphi_{\lambda}(\alpha)}=y_{\varphi_{\lambda}(\alpha)}\right) .
$$

Hence, for all $\lambda \geq \kappa_{\alpha}$ we have $z_{\varphi_{\lambda}(\alpha)}=u_{\varphi_{\lambda}(\alpha)}=w_{\varphi_{\lambda}(\alpha)}$. On the other hand the net $\left\{\left(u_{\varphi_{\lambda}(\alpha)}\right)_{\alpha \in \Gamma}\right\}_{\lambda \in \Lambda}$ has a convergent subnet like $\left\{\left(u_{\varphi_{\lambda_{\theta}}(\alpha)}\right)_{\alpha \in \Gamma}\right\}_{\theta \in T}$ to a point of $\mathcal{X}$, say $\left(v_{\alpha}\right)_{\alpha \in \Gamma}$, since $\mathcal{X}$ is compact. For all $\alpha \in \Gamma$ there exists $\theta_{\alpha} \in T$ such that $\lambda_{\theta_{\alpha}} \geq \mathcal{K}_{\alpha}$, and moreover

$$
\forall \theta \geq \theta_{\alpha}\left(u_{\varphi_{\lambda_{\theta}}(\alpha)}=v_{\alpha}\right) .
$$

Note that for all $\theta \geq \theta_{\alpha}$ we have $\lambda_{\theta} \geq \kappa_{\alpha}$, leads us to:

$$
\forall \theta \geq \theta_{\alpha}\left(z_{\varphi_{\lambda_{\theta}}(\alpha)}=v_{\alpha}=w_{\varphi_{\lambda_{\theta}}(\alpha)}\right) .
$$

Hence

$$
\lim _{\theta \in T} \sigma_{\varphi_{\lambda_{\theta}}}\left(\left(z_{\alpha}\right)_{\alpha \in \Gamma}\right)=\lim _{\theta \in T} \sigma_{\varphi_{\lambda_{\theta}}}\left(\left(w_{\alpha}\right)_{\alpha \in \Gamma}\right) \quad \text { and } \quad\left(\left(z_{\alpha}\right)_{\alpha \in \Gamma},\left(w_{\alpha}\right)_{\alpha \in \Gamma}\right) \in P(\mathcal{H}, \mathcal{X}) .
$$

2) Now suppose $\left(\left(x_{\alpha}\right)_{\alpha \in \Gamma},\left(y_{\alpha}\right)_{\alpha \in \Gamma}\right) \in L(\mathcal{H}, \mathcal{X})$ and $\left(\left(s_{\alpha}\right)_{\alpha \in \Gamma},\left(t_{\alpha}\right)_{\alpha \in \Gamma}\right)$ is an element of $\overline{\mathcal{H}\left(\left(z_{\alpha}\right)_{\alpha \in \Gamma},\left(w_{\alpha}\right)_{\alpha \in \Gamma}\right)}$. There exists a net $\left\{\sigma_{\varphi_{\lambda}}\right\}_{\lambda \in \Lambda}$ in $\mathcal{H}$, with

$$
\left(\left(s_{\alpha}\right)_{\alpha \in \Gamma},\left(t_{\alpha}\right)_{\alpha \in \Gamma}\right)=\lim _{\lambda \in \Lambda} \sigma_{\varphi_{\lambda}}\left(\left(z_{\alpha}\right)_{\alpha \in \Gamma},\left(w_{\alpha}\right)_{\alpha \in \Gamma}\right)=\lim _{\lambda \in \Lambda}\left(\left(z_{\varphi_{\lambda}(\alpha)}\right)_{\alpha \in \Gamma,}\left(w_{\varphi_{\lambda}(\alpha)}\right)_{\alpha \in \Gamma}\right) .
$$


On the other hand the net $\left\{\left(\left(x_{\varphi_{\lambda}(\alpha)}\right)_{\alpha \in \Gamma},\left(y_{\varphi_{\lambda}(\alpha)}\right)_{\alpha \in \Gamma}\right)\right\}_{\lambda \in \Lambda}$ has a convergent subnet in compact space $\mathcal{X} \times \mathcal{X}$, without loss of generality we may suppose $\left\{\left(\left(x_{\varphi_{\lambda}(\alpha)}\right)_{\alpha \in \Gamma},\left(y_{\varphi_{\lambda}(\alpha)}\right)_{\alpha \in \Gamma}\right)\right\}_{\lambda \in \Lambda}$ itself converges to a point of $\mathcal{X} \times \mathcal{X}$ like $\left(\left(m_{\alpha}\right)_{\alpha \in \Gamma},\left(n_{\alpha}\right)_{\alpha \in \Gamma}\right)$. Hence

$$
\left(\left(m_{\alpha}\right)_{\alpha \in \Gamma},\left(n_{\alpha}\right)_{\alpha \in \Gamma}\right) \in \overline{\mathcal{H}\left(\left(x_{\alpha}\right)_{\alpha \in \Gamma},\left(y_{\alpha}\right)_{\alpha \in \Gamma}\right)} \subseteq P(\mathcal{H}, \mathcal{X}) .
$$

Now for $\alpha \in \Gamma$ there exists $\kappa \in \Lambda$ such that:

$$
\forall \lambda \geq \kappa\left(\left(m_{\alpha}, n_{\alpha}\right)=\left(x_{\varphi_{\lambda}(\alpha)}, y_{\varphi_{\lambda}(\alpha)}\right)\right) .
$$

Hence we have:

$$
\begin{aligned}
m_{\alpha} \neq n_{\alpha} & \Rightarrow\left(\forall \lambda \geq \kappa\left(x_{\varphi_{\lambda}(\alpha)} \neq y_{\varphi_{\lambda}(\alpha)}\right)\right) \\
& \Rightarrow\left(\forall \lambda \geq \kappa\left(z_{\varphi_{\lambda}(\alpha)}=q \wedge w_{\varphi_{\lambda}(\alpha)}=p\right)\right) \\
& \Rightarrow \lim _{\lambda \in \Lambda} z_{\varphi_{\lambda}(\alpha)}=q \wedge \lim _{\lambda \in \Lambda} w_{\varphi_{\lambda}(\alpha)}=p \\
& \Rightarrow\left(s_{\alpha}, t_{\alpha}\right)=(q, p)
\end{aligned}
$$

and

$$
\begin{aligned}
m_{\alpha}=n_{\alpha} & \Rightarrow\left(\forall \lambda \geq \kappa\left(x_{\varphi_{\lambda}(\alpha)}=y_{\varphi_{\lambda}(\alpha)}\right)\right) \\
& \Rightarrow\left(\forall \lambda \geq \kappa\left(z_{\varphi_{\lambda}(\alpha)}=w_{\varphi_{\lambda}(\alpha)}\right)\right) \\
& \Rightarrow s_{\alpha}=\lim _{\lambda \in \Lambda} z_{\varphi_{\lambda}(\alpha)}=\lim _{\lambda \in \Lambda} w_{\varphi_{\lambda}(\alpha)}=t_{\alpha} \\
& \Rightarrow s_{\alpha}=t_{\alpha} .
\end{aligned}
$$

Hence for $\left(v_{\alpha}\right)_{\alpha \in \Gamma}:=\left(s_{\alpha}\right)_{\alpha \in \Gamma}$, we have:

$$
s_{\alpha}=\left\{\begin{array}{ll}
q, & m_{\alpha} \neq n_{\alpha}, \\
v_{\alpha}, & m_{\alpha}=n_{\alpha},
\end{array} \quad \text { and } \quad t_{\alpha}= \begin{cases}p, & m_{\alpha} \neq n_{\alpha} \\
v_{\alpha}, & m_{\alpha}=n_{\alpha} .\end{cases}\right.
$$

Using 1), $\left(\left(m_{\alpha}\right)_{\alpha \in \Gamma},\left(n_{\alpha}\right)_{\alpha \in \Gamma}\right) \in P(\mathcal{H}, \mathcal{X})$ and (4.1) we have $\left(\left(s_{\alpha}\right)_{\alpha \in \Gamma},\left(t_{\alpha}\right)_{\alpha \in \Gamma}\right) \in P(\mathcal{H}, \mathcal{X})$, which completes the proof.

Lemma 4.2. We have:

$$
L(\mathcal{H}, \mathcal{X}) \subseteq\left\{\left(\left(x_{\alpha}\right)_{\alpha \in \Gamma},\left(y_{\alpha}\right)_{\alpha \in \Gamma}\right) \in \mathcal{X} \times \mathcal{X}:\left\{\gamma \in \Gamma: x_{\gamma} \neq y_{\gamma}\right\} \text { is finite }\right\}
$$

Proof. Consider $\left(x_{\alpha}\right)_{\alpha \in \Gamma,}\left(y_{\alpha}\right)_{\alpha \in \Gamma} \in \mathcal{X}$ such that $B:=\left\{\alpha \in \Gamma: x_{\alpha} \neq y_{\alpha}\right\}$ is infinite. Choose distinct $p, q \in X$ and let:

$$
z_{\alpha}:= \begin{cases}q, & \alpha \in B \\ p, & \alpha \notin B\end{cases}
$$


By Lemma 4.1, if $\left(\left(x_{\alpha}\right)_{\alpha \in \Gamma},\left(y_{\alpha}\right)_{\alpha \in \Gamma}\right) \in L(\mathcal{H}, \mathcal{X})$, then $\left(\left(z_{\alpha}\right)_{\alpha \in \Gamma},(p)_{\alpha \in \Gamma}\right) \in L(\mathcal{H}, \mathcal{X})$. We show $\left((q)_{\alpha \in \Gamma},(p)_{\alpha \in \Gamma}\right) \in \overline{\mathcal{H}\left(\left(z_{\alpha}\right)_{\alpha \in \Gamma},(p)_{\alpha \in \Gamma}\right)}$. Suppose $U$ is an open neighbourhood of $\left((q)_{\alpha \in \Gamma},(p)_{\alpha \in \Gamma}\right)$, then there exists distinct $\alpha_{1}, \cdots, \alpha_{n} \in \Gamma$ such that for:

$$
V_{\alpha}=\left\{\begin{array}{ll}
\{q\}, & \alpha=\alpha_{1}, \cdots, \alpha_{n}, \\
X, & \alpha \neq \alpha_{1}, \cdots, \alpha_{n},
\end{array} \quad \text { and } \quad W_{\alpha}=\{p\}, \quad(\forall \alpha \in \Gamma),\right.
$$

we have

$$
\prod_{\alpha \in \Gamma} V_{\alpha} \times \prod_{\alpha \in \Gamma} W_{\alpha} \subseteq U
$$

Since $B$ is infinite, we could choose distinct $\beta_{1}, \cdots, \beta_{n} \in B$ such that $\left\{\alpha_{1}, \cdots, \alpha_{n}\right\} \cap$ $\left\{\beta_{1}, \cdots, \beta_{n}\right\}=\varnothing$. Define $\psi: \Gamma \rightarrow \Gamma$ by

$$
\psi(\alpha):= \begin{cases}\alpha_{i}, & \alpha=\beta_{i}, \quad i=1, \cdots, n, \\ \beta_{i,} & \alpha=\alpha_{i}, \quad i=1, \cdots, n, \\ \alpha, & \text { otherwise, }\end{cases}
$$

then $\psi: \Gamma \rightarrow \Gamma$ is bijective, $\sigma_{\psi} \in \mathcal{H}$ and

$$
\sigma_{\psi}\left(\left(z_{\alpha}\right)_{\alpha \in \Gamma},(p)_{\alpha \in \Gamma}\right)=\left(\sigma_{\psi}\left(\left(z_{\alpha}\right)_{\alpha \in \Gamma}\right), \sigma_{\psi}\left((p)_{\alpha \in \Gamma}\right)\right)=\left(\left(z_{\psi(\alpha)}\right)_{\alpha \in \Gamma},(p)_{\alpha \in \Gamma}\right) \in U .
$$

Hence $\left((q)_{\alpha \in \Gamma},(p)_{\alpha \in \Gamma}\right) \in \overline{\mathcal{H}\left(\left(z_{\alpha}\right)_{\alpha \in \Gamma},(p)_{\alpha \in \Gamma}\right)}$. Since $\left((q)_{\alpha \in \Gamma},(p)_{\alpha \in \Gamma}\right) \notin P(\mathcal{H}, \mathcal{X})$, we have $\left(\left(z_{\alpha}\right)_{\alpha \in \Gamma},(p)_{\alpha \in \Gamma}\right) \notin L(\mathcal{H}, \mathcal{X})$, which leads to $\left(\left(x_{\alpha}\right)_{\alpha \in \Gamma},\left(y_{\alpha}\right)_{\alpha \in \Gamma}\right) \notin L(\mathcal{H}, \mathcal{X})$ and completes the proof.

The proof of the following lemma is similar to that of Lemma 3.1.

Lemma 4.3. For $\left(\left(x_{\alpha}\right)_{\alpha \in \Gamma,}\left(y_{\alpha}\right)_{\alpha \in \Gamma}\right) \in \mathcal{X} \times \mathcal{X}$ if $\left\{\alpha \in \Gamma: x_{\alpha} \neq y_{\alpha}\right\}$ is finite and $\left(\left(z_{\alpha}\right)_{\alpha \in \Gamma},\left(w_{\alpha}\right)_{\alpha \in \Gamma}\right) \in \overline{\mathcal{H}\left(\left(x_{\alpha}\right)_{\alpha \in \Gamma},\left(y_{\alpha}\right)_{\alpha \in \Gamma}\right)}$, then $\left\{\alpha \in \Gamma: z_{\alpha} \neq w_{\alpha}\right\}$ is finite satisfying $\operatorname{card}\left(\left\{\alpha \in \Gamma: z_{\alpha} \neq w_{\alpha}\right\}\right) \leq \operatorname{card}\left(\left\{\alpha \in \Gamma: x_{\alpha} \neq y_{\alpha}\right\}\right)$.

Proof. For $n \geq 1$, if there exists distinct $\alpha_{1}, \cdots, \alpha_{n} \in \Gamma$ with $z_{\alpha_{i}} \neq w_{\alpha_{i}}$ for $i=1, \cdots, n$, then let:

$$
U_{\alpha}:=\left\{\begin{array}{ll}
\left\{z_{\alpha}\right\}, & \alpha=\alpha_{1}, \cdots, \alpha_{n}, \\
X, & \alpha \neq \alpha_{1}, \cdots, \alpha_{n},
\end{array} \quad \text { and } \quad V_{\alpha}:= \begin{cases}\left\{w_{\alpha}\right\}, & \alpha=\alpha_{1}, \cdots, \alpha_{n}, \\
X, & \alpha \neq \alpha_{1}, \cdots, \alpha_{n} .\end{cases}\right.
$$

Thus

$$
U:=\prod_{\alpha \in \Gamma} U_{\alpha} \times \prod_{\alpha \in \Gamma} V_{\alpha}
$$

is an open neighbourhood of $\left(\left(z_{\alpha}\right)_{\alpha \in \Gamma},\left(w_{\alpha}\right)_{\alpha \in \Gamma}\right)$, and there exists bijection $\varphi: \Gamma \rightarrow \Gamma$ with

$$
\left(\sigma_{\varphi}\left(\left(x_{\alpha}\right)_{\alpha \in \Gamma}\right), \sigma_{\varphi}\left(\left(y_{\alpha}\right)_{\alpha \in \Gamma}\right)\right)=\left(\left(x_{\varphi(\alpha)}\right)_{\alpha \in \Gamma},\left(y_{\varphi(\alpha)}\right)_{\alpha \in \Gamma}\right) \in U .
$$

Hence $x_{\varphi\left(\alpha_{i}\right)}=z_{\alpha_{i}}$ and $y_{\varphi\left(\alpha_{i}\right)}=w_{\alpha_{i}}$ for all $i=1, \cdots, n$. Therefore $x_{\varphi\left(\alpha_{i}\right)} \neq y_{\varphi\left(\alpha_{i}\right)}$ for all $i=1, \cdots, n$, which leads to $\left\{\varphi\left(\alpha_{1}\right), \cdots, \varphi\left(\alpha_{n}\right)\right\} \subseteq\left\{\alpha \in \Gamma: x_{\alpha} \neq y_{\alpha}\right\}$, so $n=$ $\operatorname{card}\left(\left\{\varphi\left(\alpha_{1}\right), \cdots, \varphi\left(\alpha_{n}\right)\right\}\right) \leq \operatorname{card}\left(\left\{\alpha \in \Gamma: x_{\alpha} \neq y_{\alpha}\right\}\right)$ (note that $\varphi$ is one to one), which leads to the desired result. 
Lemma 4.4. For infinite $\Gamma$ we have:

$$
L(\mathcal{H}, \mathcal{X}) \supseteq\left\{\left(\left(x_{\alpha}\right)_{\alpha \in \Gamma},\left(y_{\alpha}\right)_{\alpha \in \Gamma}\right) \in \mathcal{X} \times \mathcal{X}:\left\{\gamma \in \Gamma: x_{\gamma} \neq y_{\gamma}\right\} \text { is finite }\right\} .
$$

Proof. Use Lemmas 4.3 and 3.3.

Theorem 4.1. We have:

$L(\mathcal{H}, \mathcal{X})= \begin{cases}\left\{\left(\left(x_{\alpha}\right)_{\alpha \in \Gamma},\left(y_{\alpha}\right)_{\alpha \in \Gamma}\right) \in \mathcal{X} \times \mathcal{X}:\left\{\gamma \in \Gamma: x_{\gamma} \neq y_{\gamma}\right\} \text { is finite }\right\}, & \Gamma \text { is infinite, } \\ \{(x, x): x \in \mathcal{X}\}, & \Gamma \text { is finite. }\end{cases}$

Proof. For infinite $\Gamma$ use Lemmas 4.2 and 4.4 , also for finite $\Gamma$ note that $P(\mathcal{H}, \mathcal{X})=\{(x, x)$ : $x \in \mathcal{X}\}$.

\section{More details}

In transformation semigroup $(S, W)$ we say a nonempty subset $D$ of $W$ is invariant if $S D:=\{s w: s \in S, w \in D\} \subseteq W$. For closed invariant subset $D$ of $W$ we may consider action of $S$ on $D$ in a natural way. For closed invariant subset $D$ of $W$ one may verify easily,

$$
P(S, D) \subseteq P(S, W), \quad Q(S, D) \subseteq Q(S, W), \quad \text { and } \quad L(S, D) \subseteq L(S, W) .
$$

Suppose $Z$ is a compact Hausdorff topological space with at least two elements, by Tychonoff's theorem $Z^{\Gamma}$ is also compact Hausdorff. Again for $\varphi: \Gamma \rightarrow \Gamma$ one may consider $\sigma_{\varphi}: Z^{\Gamma} \rightarrow Z^{\Gamma}\left(\sigma_{\varphi}\left(\left(z_{\alpha}\right)_{\alpha \in \Gamma}\right)=\left(z_{\varphi(\alpha)}\right)_{\alpha \in \Gamma}\right)$, also $\mathcal{S}:=\left\{\sigma_{\varphi}: Z^{\Gamma} \rightarrow Z^{\Gamma} \mid \varphi \in \Gamma^{\Gamma}\right\}$, and $\mathcal{H}:=\left\{\sigma_{\varphi}: Z^{\Gamma} \rightarrow Z^{\Gamma} \mid \varphi \in \Gamma^{\Gamma}\right.$ and $\varphi: \Gamma \rightarrow \Gamma$ is bijective $\}$. Then for each finite nonenpty subset $A$ of $Z, A^{\Gamma}$ is a closed invariant subset of $\left(\mathcal{S}, Z^{\Gamma}\right)\left(\right.$ resp. $\left.\left(\mathcal{H}, Z^{\Gamma}\right)\right)$ and $A$ is a discrete (and finite) subset of $Z$. But using previous sections we know about $P\left(T, A^{\Gamma}\right), Q\left(T, A^{\Gamma}\right)$, and $L\left(T, A^{\Gamma}\right)$ for $T=\mathcal{H}, \mathcal{S}$. Hence for $T=\mathcal{H}, \mathcal{S}$ by:

$$
\begin{aligned}
& \bigcup\left\{P\left(T, A^{\Gamma}\right): A \text { is a finite subset of } Z\right\} \subseteq P\left(T, Z^{\Gamma}\right), \\
& \bigcup\left\{Q\left(T, A^{\Gamma}\right): A \text { is a finite subset of } Z\right\} \subseteq Q\left(T, Z^{\Gamma}\right), \\
& \bigcup\left\{L\left(T, A^{\Gamma}\right): A \text { is a finite subset of } Z\right\} \subseteq L\left(T, Z^{\Gamma}\right),
\end{aligned}
$$

we will have more data about $P\left(T, Z^{\Gamma}\right), Q\left(T, Z^{\Gamma}\right), L\left(T, Z^{\Gamma}\right)$.

\section{References}

[1] F. Ayatollah Zadeh Shirazi and F. Ebrahimifar, On generalized shift transformation semigroups, J. Math. Anal., 9(2) (2018), 70-77.

[2] F. Ayatollah Zadeh Shirazi, N. Karami Kabir and F. Heydari Ardi, A note on shift theory, Mathematica Pannonica, 19/2 (2008), Proceedings of ITES-2007, 187-195. 
[3] F. Ayatollah Zadeh Shirazi, J. Nazarian Sarkooh and B. Taherkhani, On Devaney chaotic generalized shift dynamical systems, Studia Scientiarum Mathematicarum Hungarica, 50(4) (2013), 509-522.

[4] I. U. Bronstein, Extensions of Minimal Transformation Groups, Sitjthoff and Noordhoff, 1979.

[5] R. Ellis, Lectures on Topological Dynamics, W. A. Benjamin, New York, 1969.

[6] Gherco, A. I. Ergodic sets and mixing extensions of topological transformation semigroups, Constantin Sergeevich Sibirsky (1928-1990). Bul. Acad. Ştiinţe Repub. Mold. Mat., 2 (2003), 87-94.

[7] A. Giordano Bruno, Algebraic entropy of generalized shifts on direct products, Commun. Algebra, 38/11 (2010), 4155-4174.

[8] Sh. Glasner, Proximal flows, Lecture Notes in Mathematics 517, Springer-Verlag, Berlin 1976.

[9] J. O. Yu, The regionally regular relation, J. Chungcheong Math. Soc., 19(4) (2006), 365-373. 